Reprod. Nutr. Dévelop., 1988, 28 Suppl. $\mathrm{n}^{\circ} 1,139-140$

\title{
Analyse de la distribution des tailles de particules à différents niveaux du tube digestif
}

Elisabeth GRENET, J. ROBELIN (*)

Unité de I'Ingestion

(*) Laboratoire de la Production de Viande I.N.R.A., Theix, 63122 Ceyrat, France.

Summary. An exponential distribution model was fitted to the particle size data of samples of digesta from sheep fed lucerne hay. It was best fitted to the oesophagus and rumen samples, which contain large particles, than to the duodenum and faeces samples.

Plusieurs méthodes ont été proposées pour décrire la distribution des particules végétales dans les contenus digestifs (Kennedy, 1984). Nous avons constaté sur nos données que le modèle exponentiel de Pond et al. (1984) donnait les résultats les plus satisfaisants sur des échantillons aussi différents que les bols alimentaires, les contenus du rumen et les contenus du duodénum.

Matériel et méthodes. Des échantillons de contenus digestifs ont été prélevés sur 11 moutons portant une fistule, soit sur l'œsophage (4), soit sur le rumen (4), soit sur le duodénum (3), alimentés à volonté avec le même foin de luzerne. Les échantillons de contenu de rumen et de contenu de duodénum ont été prélevés $4 \mathrm{~h}$ après la distribution des repas. Les échantillons ont été tamisés en milieu humide (Grenet, 1984) et le poids des particules recueillies sur les tamis a été exprimé en pourcentage du poids total et cumulé.

Le modèle utilisé pour exprimer le pourcentage cumulé de particules recueillies $(\mathrm{Y})$ en fonction de la maille $\mathrm{du}$ tamis $(\mathrm{m})$ est le suivant: $Y=Y_{0} \cdot e^{-k(m-p) n}$. Dans ce modèle $Y_{0}$ est égal à la matière sèche non solubilisée dans l'eau lors du tamisage (en \% du poids total) ; les paramètres estimés : $p$, $k$ et $n$ représentent respectivement la plus petite taille de particules, le taux d'accroissement du poids des particules recueillies sur les tamis rangés par ordre décroissant de taille de maille et une caractéristique de variation du taux d'accroissement. On définit la taille moyenne des particules comme étant la valeur de la maille du tamis qui retient $50 \%$ des particules.

Résultats et discussion. Au niveau de l'œsophage et du rumen (fig. 1) une fraction importante des particules est recueillie sur les tamis de taille de maille $\geqslant 1,6 \mathrm{~mm}$ tandis que toutes les particules des contenus de duodénum et des fèces ont une taille inférieure. Le modèle de distribution exponentiel s'adapte bien à ces résultats: les valeurs estimées sont proches des valeurs mesurées (tabl. 1) pour les échantillons récoltés au niveau de l'œsophage et du rumen, les écarts étant plus importants pour les échantillons de contenus de duodénum et de fèces. Cependant les écarts maxima observés sont beaucoup plus petits que les valeurs critiques indiquées par le test d'ajustement de Komolgorov-Smirnov. Le pourcentage de la variance expliquée est élevé (95 à $99 \%$ ) et les écarts-types résiduels 
sont relativement faibles ( 2 à $7 \%$ ). En définitive, le coefficient $k$ est de 0,002 , $0,02,0,02$ et 0,03 et la taille moyenne est de $1600,500,50$ et $160 \mu \mathrm{m}$ respectivement pour les bols de l'œesophage, les contenus de rumen, les contenus de duodénum et les fèces. Les particules fécales ont une taille supérieure aux particules duodénales par suite de la disparition de petites particules digestibles dans l'intestin.

En conclusion, ce modèle exponentiel s'adapte mieux aux échantillons prélevés au niveau de l'œsophage et du rumen qu'au niveau intestinal.

TABL. 1. - Pourcentages cumulés des particules (en poids de matière sèche) recueillies sur les différents tamis: valeurs estimées d'après le modèle exponentiel et différences avec les valeurs mesurées entre parenthèses.

\begin{tabular}{|c|c|c|c|c|c|c|c|c|c|c|}
\hline & \multicolumn{10}{|c|}{ Maille des tamis $(\mathrm{mm})$} \\
\hline & 8 & 6,3 & 4 & 2,5 & 1,6 & 0,8 & 0,4 & 0,16 & 0,10 & 0,05 \\
\hline Esophage & $\begin{array}{c}20,6 \\
(+1,9)\end{array}$ & $\begin{array}{c}25,1 \\
(-2,0)\end{array}$ & $\begin{array}{c}33,7 \\
(-0,8)\end{array}$ & $\begin{array}{c}42,5 \\
(+2,5)\end{array}$ & $\begin{array}{c}49,0 \\
(-1,2)\end{array}$ & $\begin{array}{c}57,4 \\
(+0,2)\end{array}$ & $\begin{array}{c}63,3 \\
(-1,7)\end{array}$ & $\begin{array}{c}68,2 \\
(-0,7)\end{array}$ & & $\begin{array}{r}71,4 \\
(+1,2)\end{array}$ \\
\hline Rumen & $\begin{array}{c}12,2 \\
(+0,1)\end{array}$ & $\begin{array}{c}15,1 \\
(-2,2)\end{array}$ & $\begin{array}{c}21,2 \\
(-0,8)\end{array}$ & $\begin{array}{c}28,1 \\
(+1,8)\end{array}$ & $\begin{array}{c}34,7 \\
(-0,3)\end{array}$ & $\begin{array}{c}44,5 \\
(+1,2)\end{array}$ & $\begin{array}{c}53,1 \\
(-2,3)\end{array}$ & $\begin{array}{c}62,0 \\
(-1,7)\end{array}$ & & $\begin{array}{c}69,8 \\
(+2,0)\end{array}$ \\
\hline Duodénum & & & & & & $\begin{array}{c}6,3 \\
(+4,0)\end{array}$ & $\begin{array}{c}16.0 \\
(+1,9)\end{array}$ & $\begin{array}{c}32,6 \\
(-5,1)\end{array}$ & $\begin{array}{c}40,7 \\
(-2,6)\end{array}$ & $\begin{array}{c}50,6 \\
(+4,0)\end{array}$ \\
\hline Fèces & & & & & & $\begin{array}{c}16,6 \\
(+7,4)\end{array}$ & $\begin{array}{c}30,6 \\
(+0,3)\end{array}$ & $\begin{array}{c}50,3 \\
(-0,4)\end{array}$ & $\begin{array}{c}59,5 \\
(-0,1)\end{array}$ & $\begin{array}{c}70,7 \\
(+6,3)\end{array}$ \\
\hline
\end{tabular}

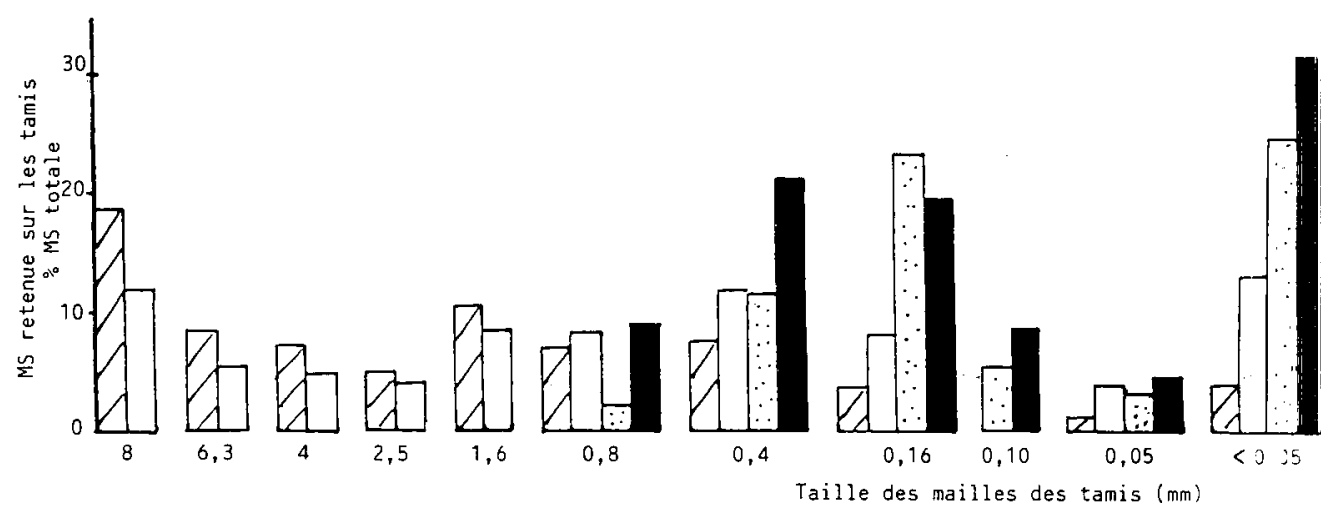

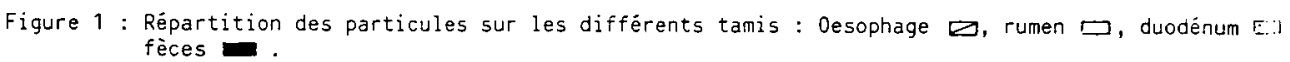

Grenet E., 1984. In P. M. Kennedy, Techniques in particle size analysis of feed and digesta in ruminants. Can. Soc. anim. Sci., Edmonton, 167.

Kennedy P. M., 1984. "Techniques in particle size analysis of feed and digesta in ruminants ". Can. J. Soc. anim. Sci., Edmonton, 195 p.

Pond K. R., Tolley E. A., Ellis W. C., Matis J. H., 1984. In P. M. Kennedy, Ed., Techniques in particle size analysis of feed and digesta in ruminants. Can. J. Soc. anim. Sci. Edmonton, 123-133. 